\title{
Electrostatic Force between two Dielectric Particles in Electrorheological Fluids: beyond Spherical Particles
}

\author{
Hongzhe TANG ${ }^{1}$, Li GAN ${ }^{1}$ and Wei $\mathrm{AN}^{2}$ \\ ${ }^{1}$ Laboratoire International Associé, Ecole Centrale de Pékin, Beihang University, Beijing 100191, China \\ ${ }^{2}$ Laboratory of Mathematics and Physics, Ecole Centrale de Pékin, Beihang University, Beijing 100191, China
}

\begin{abstract}
In an effort to increase the shear yield stress of dielectric electrorheological fluids, we focus on the electrostatic force of different forms of particles in a dielectric polarization model. By solving Laplace's equation and applying the multiple image method and the finite element method, the analytical and numerical solutions of the electrostatic force of a two-sphere structure have been studied. The results suggest that when the dielectric mismatch factor is large and when the positions of the two spheres are nearly in contact with each other, most of the analytical solutions either over- or underestimate the force. Additionally, the structure of particles beyond the spherical form is considered. Three example cases are studied to shed light on how different geometries of particles may affect the electrostatic force, thereby influencing the shear yield stress of the fluid.
\end{abstract}

\section{Nomenclature}

\begin{tabular}{|l|l|}
\hline$a, b$ & radius of two spheres, $\mathrm{m}$ \\
\hline$d$ & distance between the centers of two spheres, $\mathrm{m}$ \\
\hline $\mathbf{D}$ & electric displacement field, $\mathrm{C} \cdot \mathrm{m}^{-2}$ \\
\hline $\mathbf{E}$ & electric field, $\mathrm{V} \cdot \mathrm{m}^{-1}$ \\
\hline $\mathbf{F}$ & force, $\mathrm{N}$ \\
\hline$h$ & Planck constant, $\approx 6.626 \times 10^{-34} \mathrm{~J} \cdot \mathrm{s}$ \\
\hline$k_{\mathrm{B}}$ & Boltzmann constant, $\approx 1.381 \times 10^{-23} \mathrm{~J} \cdot \mathrm{K}^{-1}$ \\
\hline $\mathbf{p}$ & electric dipole moment, $\mathrm{C} \cdot \mathrm{m}$ \\
\hline $\mathbf{r}$ & displacement, $\mathrm{m}$ \\
\hline$\hat{\mathbf{r}}, \hat{\boldsymbol{\theta}}$ & $\begin{array}{l}\text { local orthogonal unit vectors in the directions of } \\
\text { increase } r, \theta \text { in spherical coordinate system }\end{array}$ \\
\hline$S$ & configuration entropy, $\mathrm{J} \cdot \mathrm{K}^{-1}$ \\
\hline$T$ & thermodynamic temperature, $\mathrm{K}$ \\
\hline$V$ & volume, $\mathrm{m}^{-3}$ \\
\hline$w$ & electric energy density, $\mathrm{J} \cdot \mathrm{m}^{-3}$ \\
\hline$W$ & electric potential energy, $\mathrm{J}$ \\
\hline$G r e e k$ & symbols \\
\hline$\beta$ & Clausius-Mossotti factor, $(\kappa-1) /(\kappa+2)$ \\
\hline$\gamma$ & ratio of forces between the $\mathrm{DID}$ and $\mathrm{PD}$ \\
\hline$\epsilon$ & relative permittivity \\
\hline$\epsilon_{0}$ & vacuum permittivity, $\approx 8.854 \times 10^{-12} \mathrm{~F} \cdot \mathrm{m}^{-1}$ \\
\hline$\kappa$ & dielectric mismatch factor, $\epsilon_{p} / \epsilon_{f}$ \\
\hline$\lambda$ & wavelength $\mathrm{m} ;$ scale factor \\
\hline
\end{tabular}

\begin{tabular}{|l|l|}
\hline$\rho$ & volume charge density, $\mathrm{C} \cdot \mathrm{m}^{-3}$ \\
\hline$\sigma$ & surface charge density, $\mathrm{C} \cdot \mathrm{m}^{-2}$ \\
\hline$\tau$ & spacing factor, i.e., $d / a$ \\
\hline$\phi$ & electric potential, $\mathrm{V}$ \\
\hline Superscripts \\
\hline$(L)$ & $\begin{array}{l}\text { longitudinal electric field, i.e., the applied field that is } \\
\text { parallel to the line joining the centres of the spheres }\end{array}$ \\
\hline$(T)$ & $\begin{array}{l}\text { transverse electric field, i.e., the applied field that is } \\
\text { perpendicular to the line joining the centres of the } \\
\text { spheres }\end{array}$ \\
\hline Subscripts \\
\hline$f$ & fluid \\
\hline$p$ & particle \\
\hline
\end{tabular}

\section{Introduction}

Electrorheological (ER) fluids are regarded as smart fluids, whose resistance to flow (from a liquid to a solid gel, and back) can be quickly (with response times on the order of milliseconds [1]) and dramatically altered by an applied electric field. Several applications have been proposed, including for clutches, brakes, shock absorbers, hydraulic valves, polishing, and artificial muscles [2-9]. In this work, we consider dielectric electrorheological (DER) fluids, which are made of micrometer-sized dielectric particles in an electrically insulating liquid. Compared to that of magnetorheological (MR) fluids, the yield stress of DER fluids is much lower. The shear yield stress of a general DER material is typically of several $\mathrm{kPa}$ when subjected to an electric field of $1-5 \mathrm{~V} \cdot \mathrm{mm}^{-1}$ 
[10], while the typical shear yield stress of MR fluid could vary from $2-3 \mathrm{kPa}$ with no magnetic field to 50-100 $\mathrm{kPa}$ with an applied magnetic field of $240 \mathrm{~A} \cdot \mathrm{m}^{-1}$ [11]. The DER fluid has therefore found limited use in the mechanical industry.

Our aim is to increase its yield stress, which, at the micro level, means to increase the interparticle force (mainly the electrostatic force, and other forces are discussed in Refs. [12-14]) according to the dielectric polarization model (DPM), where the electrical response is governed by linear electrostatics. Recently, dielectric effects have gained increased attention in other fields. For instance, E. Luijten et al. investigated polarization effects in charge-stabilized dielectric colloidal suspensions. They introduced an efficient technique to resolve polarization charges in dynamical dielectric geometries and demonstrated that dielectric effects qualitatively alter the predicted self-assembled structures $[15,16]$.

In this work, we ignore temperature effects and entropic effects, i.e., the first and the third term in the expression of the free energy $W[17,18]$

$$
W=\frac{3}{2} N k_{\mathrm{B}} T-\frac{1}{2} \int_{\text {particles }}\left(\mathbf{E D}-\mathbf{E}_{0} \mathbf{D}_{0}\right) d^{3} \mathbf{r}-T S,
$$

where $N$ is the total number of dielectric particles in the DER fluid; $k_{\mathrm{B}}$ is the Boltzmann constant; $T$ is the thermodynamic temperature, which is usually the room temperature; and $\mathbf{E}, \mathbf{D}\left(\mathbf{E}_{0}, \mathbf{D}_{0}\right)$ are, respectively, the local electric field and electric displacement with and without the dielectric particles. $S$ is the configuration entropy, which can be well-approximated by

$$
S=N k_{\mathrm{B}} \ln \left(\frac{\Omega}{N \lambda_{\mathrm{th}}^{3}}\right),
$$

where $\Omega$ is the volume available to the many-particle system and the thermal de Broglie wavelength is $\lambda_{\text {th }}=h / \sqrt{3 m k_{\mathrm{B}} T} \cdot h$ is the Planck constant. Consider a spherical particle at $300 \mathrm{~K}$ with a radius of $5 \mu \mathrm{m}$ and a density of $4 \mathrm{~g} \cdot \mathrm{cm}^{-3}$. The thermal wavelength is $\lambda_{\text {th }} \approx 4.11 \times 10^{-18} \mathrm{~m}$, which is very small compared to the size of the particle. Generally, the particle diameters range from 0.1 to $100 \mu \mathrm{m}$ [13]. In fact, the electrostatic energy usually dominates the thermal and entropic effects [19], i.e., the electrostatic energy of a particle is much greater than $k_{\mathrm{B}} T$ and $k_{B} T \ln \left(\Omega /\left(N \lambda_{\text {th }}^{3}\right)\right)$.

In the dipole DPM, the interparticle force depends on the electric dipole moment. Thus, it has inspired us to focus on the hierarchical structure of the particles (stick, lamella, needle-like, etc.) assembled at different scales. Charges polarized in an electric field tend to concentrate at certain positions of the particles surface so that the charges can be used efficiently to increase the shear yield stress. By optimizing the hierarchical structure combination, a high performance electrorheological fluid can be efficiently fabricated. To the best of our knowledge, work of such nature has not yet been performed. Various investigations, such as of particle patterns, arrangement structures, surface/core dielectric properties and interface structures, could be conducted to characterize the fluids. With a better characterization of electrorheological fluids, they may find wider applications in various domains, such as aviation, noise control, and automobile engineering [20].

In previous papers, the separation of variables and multiple image methods are used to determine the analytical formula of the electrostatic force between two spherical particles in the DPM [21-23]. In this paper, by proposing to investigate the problem via the finite element method (FEM) approach, we are no longer confined by the regular shape of the particles and the other parameters that we study.

In the first part, we considered the rudimentary case of the model, i.e., the point-dipole (PD) model. Then, in the following section, we consider models beyond the PD case. Next, we proceed to consider three special cases beyond the basic form as a demonstration, which provides several ideas to increase the shear yield stress in the experiment.

\section{Solution in the point-dipole model}



Figure 1. Model of two dielectric spheres in DER fluids.

Based on Sect. 4.4 of Ref. [24], we derive the formula of the electrostatic force in the point-dipole model in a completely rigorous way. As shown in Figure 1, we consider two uncharged dielectric spheres $\mathcal{A}$ and $\mathcal{B}$, of radius $a$, having identical dielectric constants $\epsilon_{0} \epsilon_{p}$, fixed in place in a liquid, with a dielectric constant $\epsilon_{0} \epsilon_{f}$, far from the electrodes so that it appears to be in a uniform electric field $\mathbf{E}=E_{0} \hat{\mathbf{z}}$. We assume $\mathcal{A}$ and $\mathcal{B}$ as two electric dipoles: $\mathcal{A}$ is at the origin, with a dipole moment $\mathbf{p}_{1} ; \mathcal{B}$, with a dipole moment $\mathbf{p}_{2}$, has a displacement $\mathbf{r}=d \hat{\mathbf{r}}$ from $\mathcal{A}$. Additionally, we assume the two particles are independent of each other. We note $\mathbf{E}_{1}$ the electric field generated by $\mathcal{A}$. The force $\mathbf{F}$ that $\mathcal{A}$ exerts on $\mathcal{B}$ can be written as

$$
\mathbf{F}_{\mathcal{A} \rightarrow \mathcal{B}}=-\nabla W=\nabla\left(\mathbf{p}_{2} \cdot \mathbf{E}_{1}\right) .
$$

$\mathbf{p}_{2}$ and $\mathbf{E}_{1}$ may be calculated by studying the electrostatic problem of particle $\mathcal{A}$. Since there are no free charges $(\rho=0)$ in the exterior or interior of the sphere, the potential around $\mathcal{A}$, noted $\phi$, satisfies Laplace's equation

$$
\nabla^{2} \phi=-\frac{\rho}{\epsilon}=0,
$$

with the boundary conditions 


$$
\left\{\begin{array}{c}
\left.\phi_{\text {in }}\right|_{r \rightarrow 0}<\infty, \\
\left.\phi_{\text {out }}\right|_{r \rightarrow \infty}=-E_{0} r \cos \theta, \\
\text { Tangentiel } \mathbf{E}:-\left.\frac{1}{a} \frac{\partial \phi_{\text {in }}}{\partial \theta}\right|_{r=a}=-\left.\frac{1}{a} \frac{\partial \phi_{\text {out }}}{\partial \theta}\right|_{r=a}, \\
\text { Normal D : }-\left.\epsilon_{p} \frac{\partial \phi_{\text {in }}}{\partial r}\right|_{r=a}=-\left.\epsilon_{f} \frac{\partial \phi_{\text {out }}}{\partial r}\right|_{r=a} .
\end{array}\right.
$$

By separation of variables and azimuthal symmetry, the general solution, called the harmonic function, can be written in the form of Legendre polynomials, and the solution of this problem can be found using the method of undetermined coefficients. We note the dielectric mismatch factor $\kappa=\epsilon_{p} / \epsilon_{f}$. The potential is therefore

$$
\left\{\begin{array}{c}
\phi_{\text {in }}=-\left(\frac{3}{\kappa+2}\right) E_{0} r \cos \theta, \\
\phi_{\text {out }}=-E_{0} r \cos \theta+\beta E_{0} \frac{a^{3}}{d^{2}} \cos \theta,
\end{array}\right.
$$

where the Clausius-Mossotti factor is $\beta=\frac{\kappa-1}{\kappa+2}$.

Then, we determine the relation between the potential and the dipole moment

$$
\phi(\mathbf{r})=\sum_{i} \frac{q_{i}}{4 \pi \epsilon_{0} \epsilon_{f}} \frac{1}{\left|\mathbf{r}-\mathbf{r}_{i}\right|} \approx \sum_{i} \frac{q_{i}}{4 \pi \epsilon_{0} \epsilon_{f}}\left(\frac{1}{d}-\mathbf{r}_{i} \cdot \nabla\left(\frac{1}{d}\right)\right)=\frac{1}{4 \pi \epsilon_{0} \epsilon_{f}} \frac{\mathbf{p} \cdot \hat{\mathbf{r}}}{d^{2}} .
$$

Hence,

$$
\phi_{\text {out }}=-E_{0} r \cos \theta+\frac{1}{4 \pi \epsilon_{0} \epsilon_{f}} \frac{\mathbf{p}_{2} \cdot \hat{\mathbf{r}}}{d^{2}}
$$

and

$$
\mathbf{p}_{2}=4 \pi \epsilon_{0} \epsilon_{f} a^{3} \beta \mathbf{E}=\mathbf{p}_{1}:=\mathbf{p}=p \hat{\mathbf{z}} .
$$

After determining $\mathbf{p}_{2}, \mathbf{E}_{1}$ may be calculated using

$\mathbf{E}_{1}=-\nabla\left(\phi_{\text {out }}+E_{0} r \cos \theta\right)=\frac{1}{4 \pi \epsilon_{0} \epsilon_{f}}\left(\frac{3(\mathbf{p} \cdot \hat{\mathbf{r}}) \hat{\mathbf{r}}-\mathbf{p}}{d^{3}}\right)-\frac{1}{3 \epsilon_{0} \epsilon_{f}} \mathbf{p} \delta^{3}(\mathbf{r})$,

where $\delta^{3}(\cdot)$ is the 3 -dimensional Dirac delta function. Since we study the case outside of $\mathcal{A}$, i.e., $\mathbf{r} \neq 0$, we have

$$
\mathbf{E}_{1}=\frac{1}{4 \pi \epsilon_{0} \epsilon_{f}}\left(\frac{3(\mathbf{p} \cdot \hat{\mathbf{r}}) \hat{\mathbf{r}}-\mathbf{p}}{d^{3}}\right) .
$$

Eventually, we obtain the expression of the force $\mathbf{F}$ that $\mathcal{A}$ exerts on $\mathcal{B}$ and the dimensionless force $\tilde{\mathbf{F}}_{\mathcal{A} \rightarrow \mathcal{B}}$.

$$
\begin{aligned}
& \mathbf{F}_{\mathcal{A} \rightarrow \mathcal{B}}=\frac{3 p^{2}}{4 \pi \epsilon_{0} \epsilon_{f} d^{4}}\left[\left(1-3 \cos ^{2} \theta\right) \hat{\mathbf{r}}-\sin 2 \theta \hat{\boldsymbol{\theta}}\right], \\
& \tilde{\mathbf{F}}_{\mathcal{A} \rightarrow \mathcal{B}}:=\frac{\mathbf{F}_{\mathcal{A} \rightarrow \mathcal{B}}}{4 \pi \epsilon_{0} \epsilon_{f}\left(E_{0} a\right)^{2}}=3 \beta^{2} \tau^{-4}\left[\left(1-3 \cos ^{2} \theta\right) \hat{\mathbf{r}}-\sin 2 \theta \hat{\boldsymbol{\theta}}\right],
\end{aligned}
$$

where $\tau=d / a$.

Specially, (i) when $\theta=0$, the electric field is parallel to the line joining the centers of the spheres, and $\tilde{\mathbf{F}}_{\mathcal{A} \rightarrow \mathcal{B}}^{(L)}=-6 \beta^{2} \tau^{-4}$. The negative value denotes attraction;

(ii) when $\theta=\frac{\pi}{2}$, the electric field is perpendicular to the line joining the centers of the spheres, and $\tilde{\mathbf{F}}_{\mathcal{A} \rightarrow \mathcal{B}}^{(T)}=3 \beta^{2} \tau^{-4}$. The positive value represents repulsion; (iii) when two spheres are in direct contact with each other, $\tilde{\mathbf{F}}_{\mathcal{A} \rightarrow \mathcal{B}}=\frac{3}{16} \beta^{2}\left[\left(1-3 \cos ^{2} \theta\right) \hat{\mathbf{r}}-\sin 2 \theta \hat{\boldsymbol{\theta}}\right]$, and the two cases above become $-\frac{3}{8} \beta^{2}$ and $\frac{3}{16} \beta^{2}$, respectively, which are both limited values.

Additionally, according to the DPM, assuming the pairwise additivity and only nearest neighbor interactions, the yield stress varies as $E_{0}^{b}$, where $E_{0}$ is the scalar quantity of the external uniform electric field, and $b \approx 2$ is commonly observed for low to moderate field strengths. At large field strengths, $b$ can decrease below 2 over a range in $\kappa=\epsilon_{p} / \epsilon_{f}(3-500)$ [13], which can be explained by the nonlinear conduction [25].

\section{Solution beyond the point-dipole model}

Beyond the dipole approximation, to solve the problem of two dielectric spheres completely, the exact analytical solution can be calculated in bispherical coordinates, which is a coordinate system in which the separation of variables is possible [21].

A much easier method is to apply the multiple image method to attain the multipole-induced-dipole (MID) model. The nomenclature is similar to that of the pointdipole model. In this case, however, we consider $\mathcal{A}$ and $\mathcal{B}$ with different radii $a$ and $b$ and different ClausiusMossotti factors $\beta_{a}$ and $\beta_{b}$, respectively. Note $d$, the distance between the centers of two spheres. Additionally, we no longer assume that the two particles are independent of each other. In Ref. [23], Siu et al. give the expression of the dipole moment of sphere $\mathcal{A}$ for two special cases: a transverse electric field $(T)$ and a longitudinal electric field $(L)$. Here, we introduce a feature parameter

$$
\lambda=\left\{\begin{array}{cl}
-1 & , \text { for }(T) \text { case } \\
2 & , \text { for }(L) \text { case }
\end{array}\right.
$$

Therefore, the expression of the dipole moment can be written in a unified form

$$
p_{\mathcal{A}}^{(T) \text { or }(L)}=(\sinh \alpha)^{3} \sum_{n=1}^{\infty}\left\{\frac{p_{A \mathcal{A}} b^{3}\left(\lambda \beta_{a}\right)^{n-1}\left(\lambda \beta_{b}\right)^{n-1}}{[b \sinh n \alpha+a \sinh (n-1) \alpha]^{3}}+\frac{p_{\mathcal{B} 0} a^{3}\left(\lambda \beta_{a}\right)^{n}\left(\lambda \beta_{b}\right)^{n-1}}{(d \sinh n \alpha)^{3}}\right\},
$$

where $p_{\mathcal{A} 0}$ and $p_{\mathcal{B} 0}$ are scalar quantities of the dipole moments in the point-dipole model

$$
p_{\mathcal{A} 0}=4 \pi \epsilon_{0} \epsilon_{f} \beta_{a} E_{0} a^{3}, p_{\mathcal{B} 0}=4 \pi \epsilon_{0} \epsilon_{f} \beta_{b} E_{0} b^{3} .
$$

The hyperbolic angle parameter $\alpha$ satisfies

$$
\cosh \alpha=\frac{d^{2}-a^{2}-b^{2}}{2 a b} \text {. }
$$

The force between the spheres is given by

$$
F^{(T)}=\frac{E_{0}}{2} \frac{\partial}{\partial d}\left(p_{\mathcal{A}}^{(T)}+p_{\mathcal{B}}^{(T)}\right), F^{(L)}=\frac{E_{0}}{2} \frac{\partial}{\partial d}\left(p_{\mathcal{A}}^{(L)}+p_{\mathcal{B}}^{(L)}\right) .
$$

The direction is along the line joining the centers of the spheres. The positive value represents repulsion, and the negative value represents attraction. 
When $n=1$, it is the point-dipole case

$$
\begin{gathered}
F_{\mathrm{PD}}^{(T) \text { or }(L)}=-12 \pi \epsilon_{0} \epsilon_{f} \lambda\left(\frac{a^{3}}{d^{2}} \beta_{a} E_{0}\right)^{2}, \\
\tilde{F}_{\mathrm{PD}}^{(T) \text { or }(L)}:=\frac{F_{\mathrm{PD}}^{(T) \text { or }(L)}}{4 \pi \epsilon_{0} \epsilon_{f}\left(E_{0} a\right)^{2}}=-3 \lambda \beta_{a}^{2}\left(\frac{a}{d}\right)^{4},
\end{gathered}
$$

which is the same as Eq. (2.10) with $\theta=0$ or $\frac{\pi}{2}$.

Considering the $n=1$ and $n=2$ terms, we obtain the expression in the dipole-induced-dipole (DID) model. The ratio $\gamma$ of the forces between the DID and the PD models can be written as

$$
\begin{aligned}
\gamma^{(T) \text { or }(L)} & :=\frac{F_{\mathrm{DID}}^{(L)}}{F_{\mathrm{PD}}^{(L)}} \text { or } \frac{F_{\mathrm{DID}}^{(T)}}{F_{\mathrm{PD}}^{(T)}} \\
& =1+\frac{\lambda \beta_{a} a^{3} d^{5}}{\left(d^{2}-b^{2}\right)^{4}}+\frac{\lambda \beta_{b} b^{3} d^{5}}{\left(d^{2}-a^{2}\right)^{4}}+\frac{\lambda^{2} \beta_{a} \beta_{b} a^{3} b^{3}\left(3 d^{2}-a^{2}-b^{2}\right)}{\left(d^{2}-a^{2}-b^{2}\right)^{4}} .
\end{aligned}
$$

More terms can be considered, but the calculation becomes much more complex. The multipole-induceddipole model includes all the terms. After extensive calculations, we obtain the expression of the electrostatic force in the MID model

$$
\begin{aligned}
\tilde{F}_{\mathrm{MID}}^{(T) \text { or }(L)} & :=\frac{F_{\mathrm{MID}}^{(T) \text { or }(L)}}{4 \pi \epsilon_{0} \epsilon_{f} a b E_{0}^{2}} \\
& =\frac{3}{2}(a b)^{2}\left\{G \sum_{n=1}^{\infty}\left[\left(\lambda^{2} \beta_{a} \beta_{b}\right)^{n}(A+B)\right]\right. \\
& \left.-\sinh ^{3} \alpha \sum_{n=1}^{\infty}\left[\left(\lambda^{2} \beta_{a} \beta_{b}\right)^{n}(C+D)\right]\right\},
\end{aligned}
$$

where

$$
\begin{aligned}
& A=-\frac{2}{(d \sinh n \alpha)^{3}}, B=\sum_{\substack{i \neq j \\
(i, j) \in\{a, b\}^{2}}} \frac{1}{\lambda^{2} \beta_{i}(i \sinh n \alpha+j \sinh (n-1) \alpha)^{3}}, \\
& C=\frac{2}{\lambda d^{2} \sinh ^{3} n \alpha}\left(\frac{1}{d^{2}}+\frac{n \cosh n \alpha}{a b \sin \alpha \sinh n \alpha}\right), \\
& D=\sum_{i \neq j} \frac{d(i n \cosh n \alpha+j(n-1) \cosh (n-1) \alpha)}{\lambda^{2} \beta_{i} a b \sinh \alpha(i \sinh n \alpha+j \sinh (n-1) \alpha)^{4}}, \\
& G=\frac{d}{2(a b)^{2}} \cosh \alpha \sqrt{\prod_{(i, j) \in\{ \pm 1\}^{2}}(d+i a+j b)} .
\end{aligned}
$$

Inspired by the PD model, the feature parameter $\lambda$ could be extended as

$$
\lambda=3 \cos ^{2} \theta-1,
$$

and $\tilde{F}_{M I D}$ can be generalized to the projection of the electrostatic force between two spheres in the direction of $-\hat{\mathbf{r}}$. Several limitations of Eq. (3.8) warrant comment. As it is an approximation in the MID model, more details will be discussed in the following part.

\section{Preliminary validation works for the solution beyond the spherical form}

So far, by confining ourselves to the hypothesis of an ideal spherical particle, there is a difference between the theoretical and experimental results: what about other forms of particle $\mathcal{A}$ ? By varying the boundary $\partial \Omega_{\mathcal{A}}$, the case is transferred to a Dirichlet problem for Laplace's equation, whose analytical solution is difficult to calculate by adding correction terms as in the case of a two-sphere problem. An alternative approach is to use a numerical solution; the finite element method (FEM) is investigated for its feasibility in approximating the solution for problems of such nature.

Many researchers have considered cases beyond a typical two-sphere model. For instance, Wen et al. considered the coated sphere structure, and shown that the use of an effective dielectric constant concept based on the first-principles method can yield quantitative predictions [19]. Tao et al. adopted an infinite chain of dielectric particles [22], while Ahn et al. investigated nsphere clusters [26]. J. G. Cao et al. considered the microstructure evolution from a random structure that consists of spherical particles to chains and then to stable lamellar patterns [27]. However, to the best of the authors' knowledge, there are few studies that consider models beyond that of spherical particles. Hence, before studying the non-spherical case, we start from the abovementioned spherical model, the simplest case, to verify its numerical solution accuracy when compared with the analytical solution.

Consider a pair of dielectric spheres $\mathcal{A}$ and $\mathcal{B}$ of identical radii $a$ and dielectric constants $\epsilon_{0} \epsilon_{p}$ being embedded in an electrically insulating liquid with dielectric constant $\epsilon_{0} \epsilon_{f}$ in response to an applied electric field, $\mathbf{E}=E_{0} \hat{\mathbf{z}}$, which is parallel to the line joining the centers of the spheres. We note the spacing factor $\tau=d / a$. Eqs. (3.7) and (3.8) can be simplified as

$$
\begin{gathered}
F_{\mathrm{DID}}^{(L)}=\gamma F_{\mathrm{PD}}^{(L)}=\left[1+\frac{4 \beta \tau^{5}}{\left(\tau^{2}-1\right)^{4}}+\frac{4 \beta^{2}\left(3 \tau^{2}-2\right)}{\left(\tau^{2}-2\right)^{4}}\right] F_{\mathrm{PD}}^{(L)}, \\
\tilde{F}_{\mathrm{DID}}^{(L)}:=\frac{F_{\mathrm{DID}}^{(L)}}{4 \epsilon_{0} \epsilon_{f}\left(E_{0} a\right)^{2}} . \\
F_{\mathrm{MID}}^{(L)}=K\left[G^{\prime} \sum_{n=1}^{\infty}\left(A^{\prime}+B^{\prime}\right)-4(\sinh \alpha)^{3} \sum_{n=1}^{\infty}\left(C^{\prime}+D^{\prime}\right)\right]:=K \sum_{n=1}^{\infty} F_{n}^{(L)}, \\
\tilde{F}_{\mathrm{MID}}^{(L)}:=\frac{F_{\mathrm{MID}}^{(L)}}{4 \epsilon_{0} \epsilon_{f}\left(E_{0} a\right)^{2}},
\end{gathered}
$$

where

$$
\begin{aligned}
& A^{\prime}=\frac{(2 \beta)^{2 n-1}}{(\sinh n \alpha+\sinh (n-1) \alpha)^{3}}, B^{\prime}=\frac{(2 \beta)^{2 n}}{(\tau \sinh n \alpha)^{3}}, \\
& C^{\prime}=\frac{(2 \beta)^{2 n-1}}{\sqrt{\tau^{2}-4}} \frac{n \cosh n \alpha+(n-1) \cosh (n-1) \alpha}{(\sinh n \alpha+\sinh (n-1) \alpha)^{4}}, \\
& D^{\prime}=\frac{(2 \beta)^{2 n}}{(\tau \sinh n \alpha)^{3}}\left(\frac{n \operatorname{coth} n \alpha}{\sqrt{\tau^{2}-4}}+\frac{1}{2 \tau}\right), \\
& G^{\prime}=\frac{\tau^{2}\left(\tau^{2}-2\right) \sqrt{\tau^{2}-4}}{2}, K=3 \pi \epsilon_{0} \epsilon_{f}\left(E_{0} a\right)^{2} .
\end{aligned}
$$

Now, we study the convergence of Eq. (4.2). We calculate $\log \left(\sum_{n=2}^{100} F_{n}^{(L)} / F_{1}^{(L)}\right)$ in four cases, which are 
shown in Figure 2. The results reveal that Eq. (4.2) is inapplicable when the two spheres are very close and $\kappa$ is large. A further analysis indicates that when either of the following occurs, Eq. (4.2) is divergent and thus inapplicable: (i) $\kappa>50$ and $\tau<2.1$, (ii) $\kappa>10$ and $\tau<2.03$, or (iii) $\kappa>4$ and $\tau<2.004$.
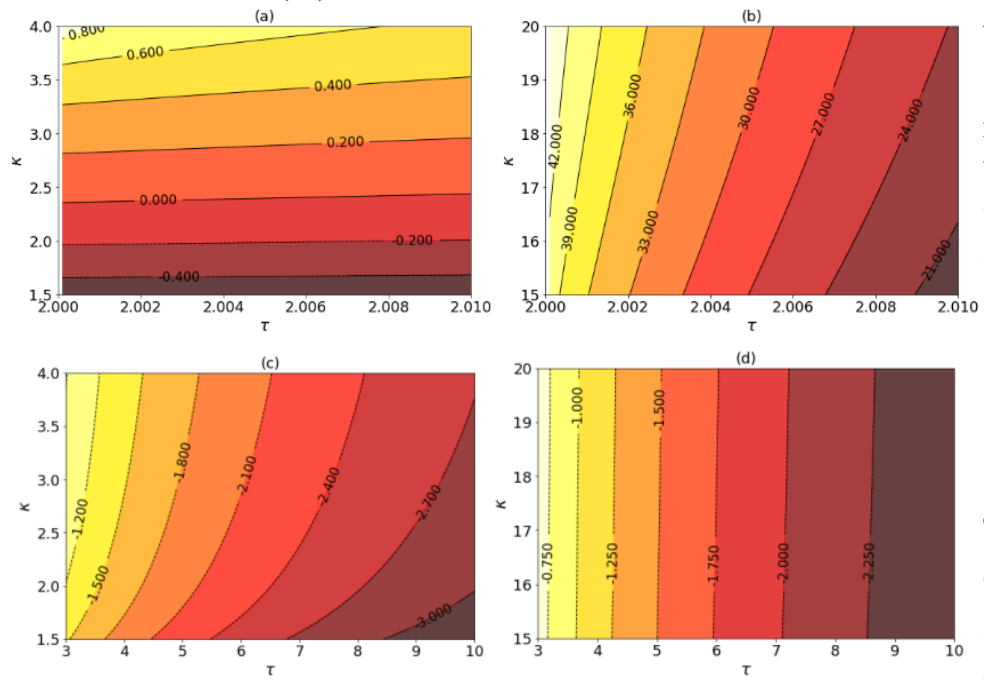

Figure 2. Contour line represents $\log \left(\sum_{n=2}^{100} F_{n}^{(L)} / F_{1}^{(L)}\right)$, as a function of the dielectric mismatch factor $\kappa=\epsilon_{p} / \epsilon_{f}$ and the spacing factor $\tau=d / a$. The solid lines represent positive values, while the dotted lines represent negative values. The values become stable when more $F_{n}^{(L)}$ terms are considered, except for the case in (b), where the values are not stable and diverge continually, since the values on the contour lines are far more than 1 and increase with the increase of $n$ (When $n$ comes to 580 , the values on the domain of (b), i.e., $(\tau, \kappa)=(2,2.01] \times[15,20]$, all exceed 100), which means Eq. (4.2) overestimates the dipole moment when the two spheres are very close and $\kappa$ is large.

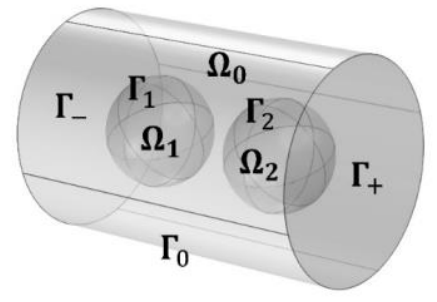

Figure 3. Schematic diagram of the model for a numerical calculation. $\Omega_{1}$ and $\Omega_{2}$ are two open bounded sets of two particles, and their boundaries $\Gamma_{1,2}=\partial \Omega_{1,2}$ are both Lipschitz continuous. $\Omega_{0}=\overline{\Omega_{1} \cup \Omega_{2}}$ is an open bounded set of the retaining cylinder. The Lipschitz-continuous boundary $\partial \Omega_{0}$ consists of the area of the top and bottom base $\Gamma_{ \pm}$, the area of the side $\Gamma_{0}$, and $\Gamma_{1,2}$.

\subsection{Model building}

To ensure the accuracy of the numerical solution, axisymmetric structures are used in the model. As shown in Figure 3, to create an environment of a uniform electric field in the cylinder, different voltages $V_{+,-}$are loaded on the top and bottom bases $\Gamma_{+,-}$. Two spheres are placed longitudinally with respect to the electric field in the cylinder. We define $a$ as the radius of sphere, $\lambda_{\text {long }} a \tau+2 a$ as the height of the cylinder, and $\lambda_{\text {pol }} a$ as the radius of the cylinder. In this paper, two parameters $\lambda_{\text {long }}$ and $\lambda_{\text {pol }}$ are introduced to help determine a suitable mesh space that would be computationally inexpensive while ensuring that the assumption of a quasi-uniform electrical space is valid, which means we choose an arbitrary $\left(\lambda_{m}, \lambda_{l}, \lambda_{p}\right)$ with a small norm from the domain $\left\{\left(\lambda_{m}, \lambda_{l}, \lambda_{p}\right) \in \mathbb{R}_{+}^{3} \mid \max _{|\mathbf{v}|=1}\left\{\nabla_{\mathbf{v}} \tilde{W}_{\mathcal{A}}\left(\lambda_{m}, \lambda_{l}, \lambda_{p} ; \beta_{0}, \tau_{0}\right)\right\}<\varepsilon_{0}\right\}$, with $\beta_{0}, \tau_{0}$, and $\varepsilon_{0}$ fixed.

\subsection{Method}

The center of $\mathcal{A}$ is fixed along the longitudinal axis, and $\mathcal{B}$ is fixed at the origin. According to the principle of virtual work and Newton's third law, the scalar quantity of the force $\mathbf{F}$ that $\mathcal{A}$ exerts on $\mathcal{B}$ can be written as

$F_{\mathcal{A} \rightarrow \mathcal{B}}=-F_{\mathcal{B} \rightarrow \mathcal{A}}=\frac{\delta W_{\mathcal{A}}}{\delta z}=\lim _{\Delta z \rightarrow 0} \frac{\int_{\mathcal{A}} w_{\mid d+\Delta z} d^{3} x-\int_{\mathcal{A}} w_{|d\rangle} d^{3} x}{\Delta z}$,

where $w_{|d\rangle}=\frac{1}{2} \epsilon_{0} \epsilon_{p}\left|\mathbf{E}_{|d\rangle}\right|^{2}=\frac{1}{2} \epsilon_{0} \epsilon_{p}\left|\nabla \phi_{|d\rangle}\right|^{2} \cdot w_{|d\rangle}, \mathbf{E}_{|d\rangle}$, and $\phi_{d\rangle}$ are, respectively, the electrostatic free energy density, the electric field and the potential function in the model where $\mathcal{A}$ is fixed at $z=d$. $\phi$ can be calculated using

$$
\nabla^{2} \phi_{i}=0, \text { in } \Omega_{i}, i=0,1,2,
$$

with the boundary conditions

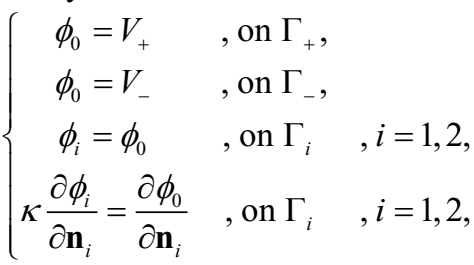

where $\mathbf{n}_{i}$ denotes the normal to the boundary $\Gamma_{i}$, whose direction is from $\Omega_{i}$ to $\Omega_{0}$, and the dielectric mismatch factor is $\kappa=\epsilon_{p} / \epsilon_{f}$.

Therefore, to determine the force, we simply calculate the distribution of the potential, where we apply a volume-discretization method, namely, the finite element method (FEM), due to its adaptability to complex geometries and its ease of handling discontinuous gradients of a variable. The basic steps of the FEM are shown in Figure 4. Based on the three-dimensional FEM in Sect. 5.3 of Ref. [28], we calculate the distribution of the potential, and therefore the electrostatic energy and the force.

To solve the problem beyond the regular form of particles and pay more attention to the post-processing, we use an unstructured grid for the FEM mesh. Tetrahedrons are employed as the cell shapes. The 
Bowyer-Watson algorithm [29, 30] is used to compute the Delaunay triangulations. The parameters of the tetrahedral mesh generated using the Delaunay tessellation method are described below. The maximum element growth rate is 1.3. The curvature factor is 0.2 . The average element quality is 0.7704 . The subdivision parameter on the $\mathrm{x}, \mathrm{y}, \mathrm{z}$-direction scale is $\lambda_{\text {mesh }}$ for $\Omega_{1,2}$ and 1 for $\Omega_{0}$. Figure 5 shows the FEM unstructured mesh used for the numerical calculation.

Solving the sparse matrix equation, the conjugate gradient method and the smoothed aggregation algebraic multigrid (SA-AMG) method are used [31], where the convergence criterion (the relative error after each iteration compared to that of the previous one) is set to be $1 \times 10^{-3}$ to ensure the result converges well.

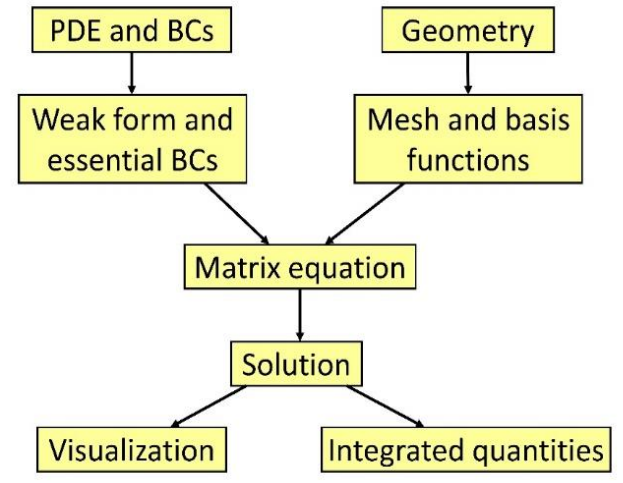

Figure 4. Basic steps to perform the finite element method (FEM) to solve a second-order partial differential equation (PDE) in a computational domain $\Omega$ with boundary conditions (BCs) on the boundary $\partial \Omega$ of the domain.
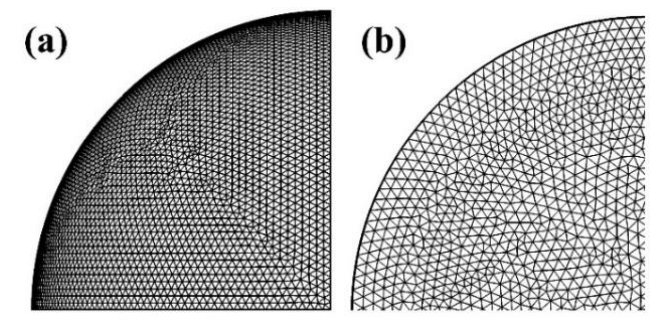

(c)



Figure 5. FEM unstructured mesh (a quarter of the whole part in view) used for the numerical calculation with $\lambda_{\text {mesh }}=20$ (a) (y-z plan) for a spherical particle $\Omega_{1}$, (b) (y-z plan) for the bottom of the cylinder, and (c) (x-y plan) for the side of the middle cylinder.

\subsection{Electrostatic energy}

The electrostatic energy of a single spherical particle in a uniform electric field can be calculated according to Eq. (1.4) as follows:

$$
\begin{gathered}
\mathbf{E}_{\mathrm{in}}=-\nabla \phi_{\mathrm{in}}=\frac{3}{\kappa+2} E_{0} \hat{\mathbf{z}}:=E_{\mathrm{in}} \hat{\mathbf{z}}, \\
\tilde{W}_{\mathcal{A}}:=\frac{W_{\mathcal{A}}}{4 \pi \epsilon_{0} \epsilon_{f} E_{0}^{2} a^{3}}=\frac{\int_{\mathcal{A}} w d^{3} x}{4 \pi \epsilon_{0} \epsilon_{f} E_{0}^{2} a^{3}}=\frac{3}{2} \frac{\kappa}{(\kappa+2)^{2}} .
\end{gathered}
$$

Since $\tilde{W}_{\mathcal{A}}^{\prime}=-\frac{3}{2} \frac{\kappa-2}{(\kappa+2)^{3}}, \tilde{W}_{\mathcal{A}}$ attains its maximum value at $\kappa=2$.

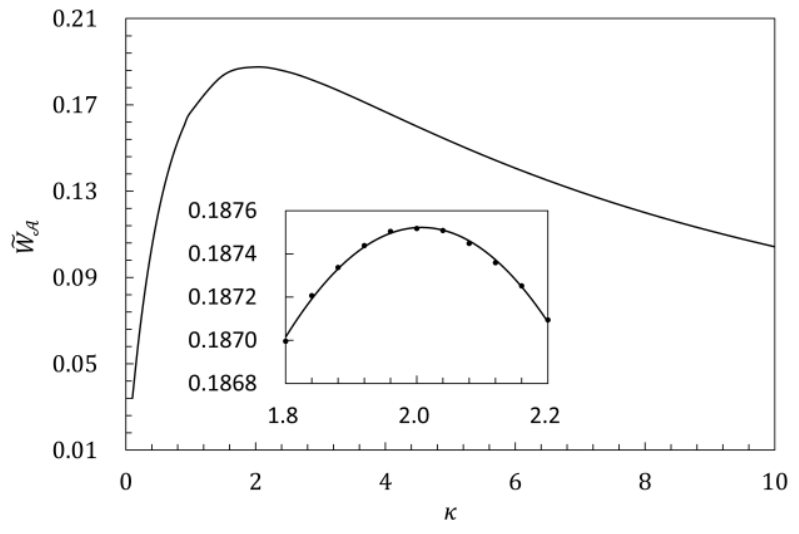

Figure 6. Electrostatic energy (in units of $\left.1 /\left(4 \pi \epsilon_{0} \epsilon_{f} E_{0}^{2} a^{3}\right)\right)$ of the sphere in the single-sphere case where we consider a dielectric sphere of radius $a$ and dielectric constant $\epsilon_{0} \epsilon_{p}$ being embedded in an electrically insulating liquid with dielectric constant $\epsilon_{0} \epsilon_{f}$ in response to an applied electric field $\mathbf{E}=E_{0} \hat{\mathbf{z}}$ as a function of the dielectric mismatch factor $\kappa=\epsilon_{p} / \epsilon_{f}$ ranging from 0.1 to 10 .

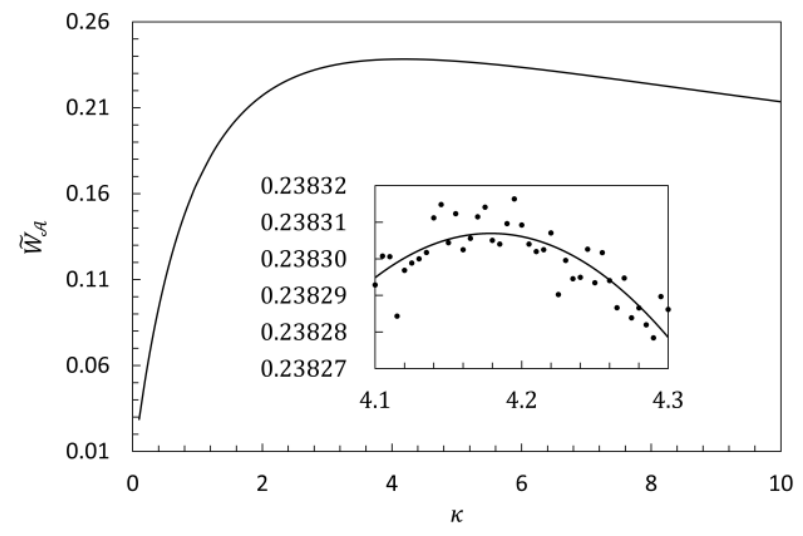

Figure 7. Electrostatic energy (in units of $\left.1 /\left(4 \pi \epsilon_{0} \epsilon_{f} E_{0}^{2} a^{3}\right)\right)$ of each sphere in the double-sphere case where we consider two identical dielectric spheres, of radius $a$ and dielectric constant $\epsilon_{0} \epsilon_{p}$ being embedded in an electrically insulating liquid with dielectric constant $\epsilon_{0} \epsilon_{f}$ in response to an applied electric field $\mathbf{E}=E_{0} \hat{\mathbf{z}}$, which is parallel to the line joining the centers of the spheres, as a function of the dielectric mismatch factor $\kappa=\epsilon_{p} / \epsilon_{f}$ ranging from 0.1 to 10 .

In the simple-sphere case, $\tau$ is defined to be 2 . As shown in Figure 6, for a series of test cases, the maximum relative error is $5.54 \times 10^{-4}$ with $\kappa=9.5$, which suggests the validity of the simulation.

As for the case of two spherical particles, the calculation becomes complex. In Figure 7, the 
dimensionless energy per sphere $\tilde{W}_{A}$ in the doublesphere case attains its maximum when $\kappa$ is between 4.10 and 4.25. A similar trend occurs in the triple-sphere case. $\tilde{W}_{\mathcal{A}}$ attains its maximum value of 0.8726 when $\kappa$ is between 7.30 and 7.70 .

\subsection{Electrostatic force for the double-sphere case}

Figure 8 presents the interaction energy of one particle $\tilde{W}_{\mathcal{A}}$ for the double-sphere case as a function of the spacing $d$ at $\kappa=1,2,3,5$, and 10. Using Eq. (3.3), we obtain the curves shown in Figure 9.

We compare the numerical simulation result with the MID one. See Figures 9a-9d. When $\tau$ tends to 2, the condition $\kappa=3$ is a criterion where the two results are approximately the same. When $\kappa<3$, Eq. (3.2) underestimates the electrostatic force, while when $\kappa>3$, Eq. (3.2) overestimates the force. When $\kappa \gg 1$ and $\tau$ tends to 2 , there is a huge difference between the numerical results and the approximated theoretical results. For example, for $\kappa=10$ and $\tau=2.05$ the MID result is $617 \%$ that of the simulation. When $\tau$ tends to 2 , all the MID results tend to infinity, which is impossible in physics, while the results from all simulation cases remain bounded.

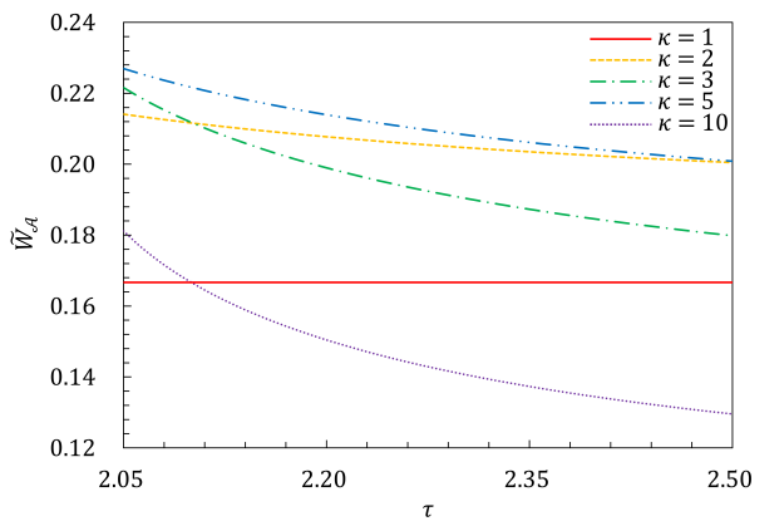

Figure 8. Electrostatic energy (in units of $\left.1 /\left(4 \pi \epsilon_{0} \epsilon_{f} E_{0}^{2} a^{3}\right)\right)$ of each sphere in the double-sphere case, where we consider two identical dielectric spheres of radius $a$ and dielectric constant $\epsilon_{0} \epsilon_{p}$ being embedded in an electrically insulating liquid with dielectric constant $\epsilon_{0} \epsilon_{f}$ in response to an applied electric field $\mathbf{E}=E_{0} \hat{\mathbf{z}}$, which is parallel to the line joining the centers of the spheres, as a function of the spacing factor $\tau=d / a$ ranging from 2.05 to 2.5 at $\kappa=1,2,3,5$, and 10 .
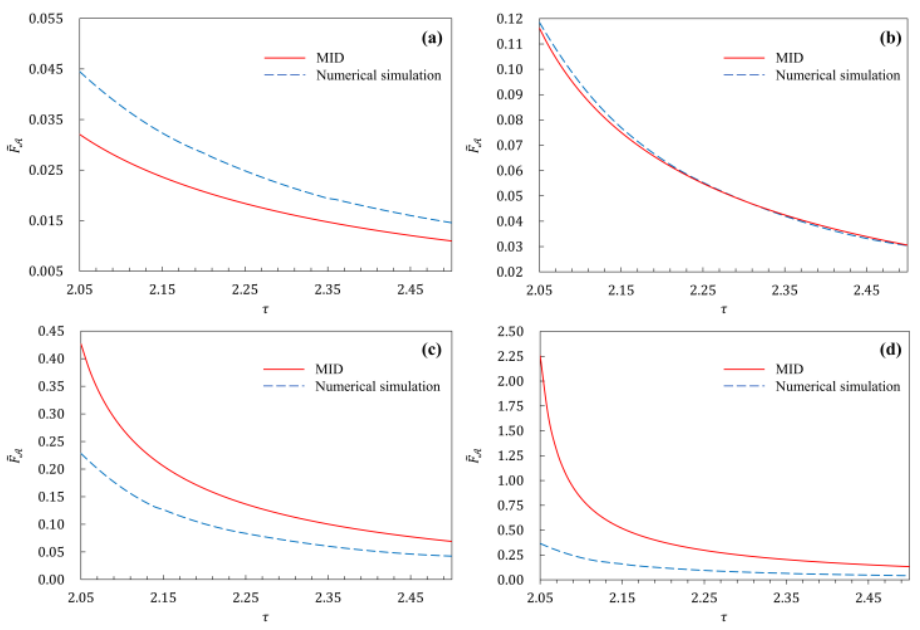

Figure 9. Electrostatic force (in units of $1 /\left(4 \epsilon_{0} \epsilon_{f} E_{0}^{2} a^{2}\right)$ )

calculated in the MID (red solid line) $v s$. the numerical simulation (blue long dash line) of one sphere exerted by the other sphere in the double-sphere case as a function of the spacing factor $\tau=d / a$ ranging from 2.05 to 2.50 at (a) $\kappa=2$, (b) $\kappa=3$, (c) $\kappa=5$, and (d) $\kappa=10$.

\section{Solution beyond the spherical form (three demonstrations)}

\subsection{Example 1: Earth-and-moon structure}

In the first example, we consider two spheres with different radii. The sum of the volumes of the spheres is fixed and equals the volume of two spheres with the same radii $a$. Note $\lambda$ is the ratio of the two radii. The radii can then be written as $\left(\frac{2 a^{3}}{1+\lambda^{3}}\right)^{1 / 3}$ and $\lambda\left(\frac{2 a^{3}}{1+\lambda^{3}}\right)^{1 / 3}$. In Figure 10, we plot the force $\tilde{F}_{\mathcal{A}}=\frac{F_{\mathcal{A}}}{4 \epsilon_{0} \epsilon_{f}\left(E_{0} a\right)^{2}}$ with different $\lambda$ at $\kappa=10$ and $d=(1+\lambda)\left(\frac{2 a^{3}}{1+\lambda^{3}}\right)^{1 / 3}$. The maximum value of $\tilde{F}_{\mathcal{A}}$ is reached when $\lambda=1$. For $\lambda>1$, $\tilde{F}_{\mathcal{A}}$ decreases sharply, which reveals that to obtain the biggest force, the particles should be as identical as possible. The difference in size will result in great changes in the electrostatic force.

Now, we consider the sub-structures that are uniformly distributed across a sphere. To create the model, we consider $N$ number of points distributed uniformly on a sphere, of which several approximate solutions have been proposed [32-34].

Consider $N=20$. Since the regular dodecahedron has 20 vertices, its circumscribed sphere, the one that touches the regular dodecahedron at all vertices, can be considered as the base sphere. As shown in Figure 11, the Cartesian coordinates of the 20 vertices of a regular dodecahedron where the radius of its circumscribed sphere is $\sqrt{3}$ can be defined as follows: 
$\left(0, \pm \frac{1}{\phi}, \pm \phi\right),\left( \pm \frac{1}{\phi}, \pm \phi, 0\right),\left( \pm \phi, 0, \pm \frac{1}{\phi}\right),( \pm 1, \pm 1, \pm 1)$, where $\phi=\frac{1+\sqrt{5}}{2}=1.6180 \cdots$ is the golden ratio.

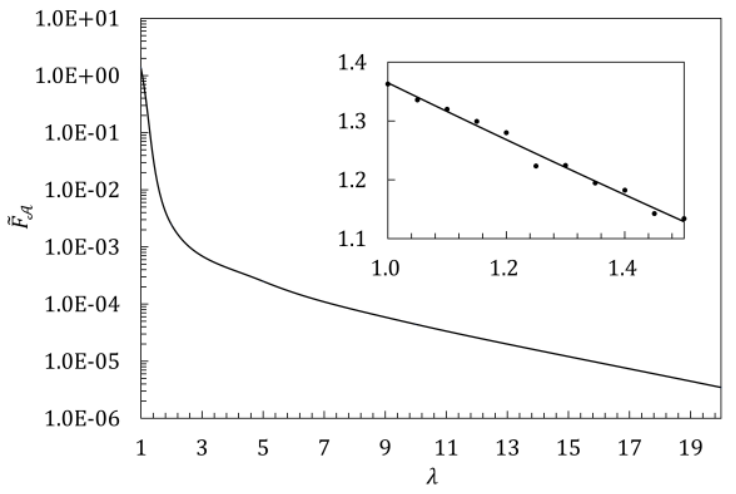

Figure 10. Semi-logarithmic plot of the electrostatic force (in units of $\left.1 /\left(4 \epsilon_{0} \epsilon_{f} E_{0}^{2} a^{2}\right)\right)$ of one earth-and-moon structure as a function of the ratio of the two radii $\lambda$ ranging from 1 to 20 at the different dielectric mismatch factor $\kappa=10$ and spacing factor $d=(1+\lambda)\left(\frac{2 a^{3}}{1+\lambda^{3}}\right)^{1 / 3}$

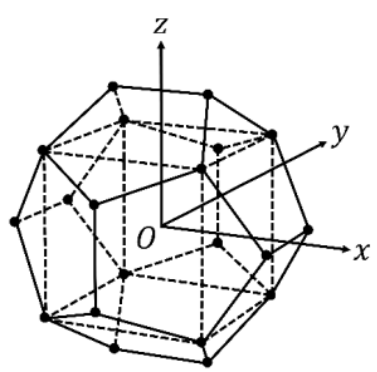

Figure 11. Regular dodecahedron in Cartesian coordinates

To rotate one of the particles, we introduce the rotation matrix. Given a unit vector $\hat{\mathbf{u}}=(x, y, z)$, the matrix for a rotation by angle $\theta$ about an axis in the direction of $\hat{\mathbf{u}}$ is

$$
\mathcal{M}(\hat{\mathbf{u}}, \theta)=\exp \left(\left[\begin{array}{ccc}
0 & -z \theta & y \theta \\
z \theta & 0 & -x \theta \\
-y \theta & x \theta & 0
\end{array}\right]\right) \text {. }
$$

In the following examples, we let $\hat{\mathbf{u}}=(x, y, z)=(0,0,1)$.

\subsection{Example 2: Litchi structure}

Consider two dielectric basic spheres $\mathcal{A}$ and $\mathcal{B}$ of identical radii $a$, with a distance $d$ between them, on which there are 20 small spheres of radii $\lambda_{\mathrm{cp}} a$ whose centers are situated on each of the 20 vertices of a regular dodecahedron as defined above for each basic sphere. We perform the rotation transformation $\mathcal{M}(\hat{\mathbf{u}}, \theta)$ for one of the basic sphere; see Figure 12. The volume of one litchistructured particle $V_{\text {cp }}$ is calculated to be $\left(15 \lambda_{\mathrm{cp}}^{4}+40 \lambda_{\mathrm{cp}}^{3}+4\right) \pi a^{3} / 3$. Consider $\lambda_{\mathrm{cp}}=0.2$. Thus, $V_{\text {cp }}=1.448 \pi a^{3}$.

Consider $\kappa=10$ and $\tau=d / a=2.1$. When $\theta=55^{\circ}$, the two particles are nearly in contact with each other. Thanks to the symmetry, only the range $55^{\circ}-90^{\circ}$ needs to be studied. Figure 13 presents the electrostatic energy $\tilde{W}_{\mathcal{A}}=\frac{W_{\mathcal{A}}}{4 \pi \epsilon_{0} \epsilon_{f} E_{0}^{2} a^{\prime 3}}$ as a function of $\theta$ ranging from $55^{\circ}$ to $90^{\circ}$, where the effective radius $a^{\prime}$ is the radius of a sphere with volume $V_{\text {cp }}$. The energy declines with the increase of the rotation angle. Starting from $74^{\circ}$, the ratio between the energy at $\theta$ and that at $90^{\circ}$ is smaller than $1 \times 10^{-3}$. Figure 14 shows the case for the rotation angle $\theta=\pi / 2$. We calculate $\tilde{F}_{\mathcal{A}}$ for the two-sphere case and the litchi structure using $\frac{F_{\mathcal{A}}}{4 \epsilon_{0} \epsilon_{f}\left(E_{0} a\right)^{2}}$ and $\frac{F_{\mathcal{A}}}{4 \epsilon_{0} \epsilon_{f}\left(E_{0} a^{\prime}\right)^{2}}$, respectively. At the position in which the two particles are just in contact with each other, $\tau$ is 2 and approximately 2.08 for the two-sphere and litchi structures, respectively. It is noted that in both cases, when the two particles are increasingly near to each other, there is a significant increase in the force $\tilde{F}_{\mathcal{A}}$, which tends to a finite value. The force at the limit position of the litchi structure is $129 \%$ that of the two-sphere case, which indicates that the litchi structure can help increase the electrostatic force.

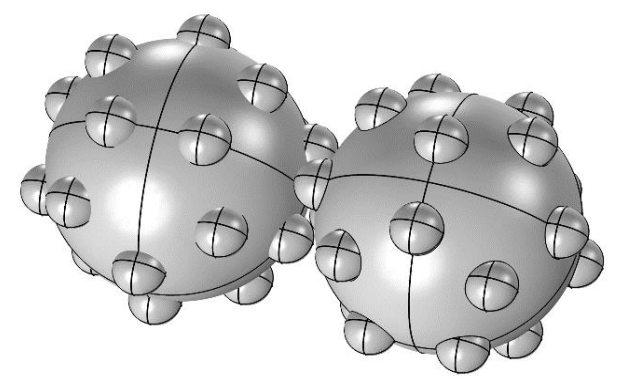

Figure 12. Schematic diagram of the litchi structure.



Figure 13. Electrostatic energy (in units of $1 /\left(4 \pi \epsilon_{0} \epsilon_{f} E_{0}^{2} a^{\prime 3}\right)$ ) of one litchi structure as a function of the rotation angle $\theta$ with 
the dielectric mismatch factor $\kappa=10$ and the spacing factor $\tau=d / a=2.1$.

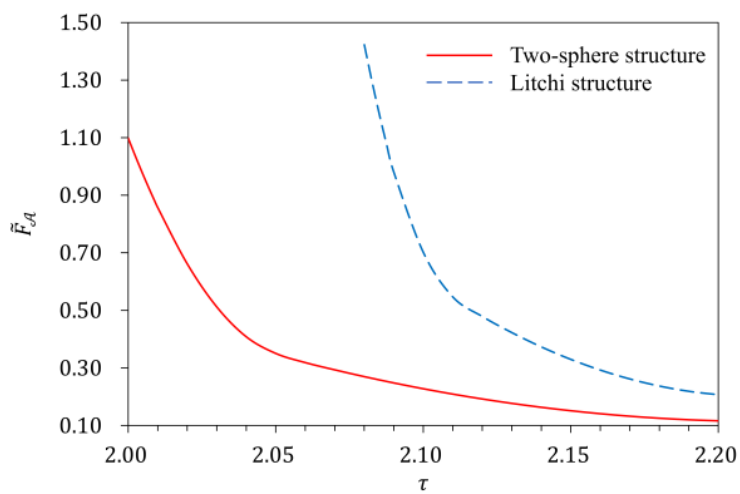

Figure 14. Electrostatic force (in units of $1 /\left(4 \epsilon_{0} \epsilon_{f} E_{0}^{2} a^{2}\right)$ for the two-sphere structure and $1 /\left(4 \epsilon_{0} \epsilon_{f} E_{0}^{2} a^{\prime 2}\right)$ for the litchi structure) as a function of the spacing factor $\tau=d / a$ with $\kappa=10$ for the two-sphere structure (red solid line) $v s$. the litchi structure (blue long dash line).

\subsection{Example 3: Sea urchin structure}

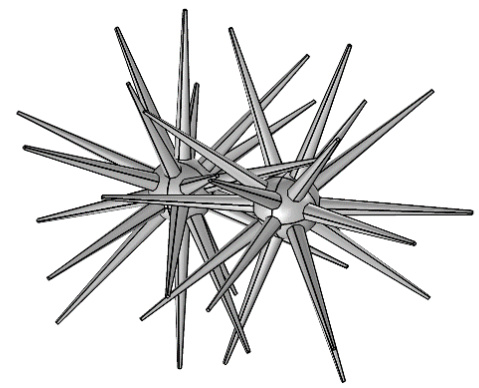

Figure 15. Schematic diagram of the sea urchin structure.

In this example, by replacing small spheres with spikes, we create a sea urchin structure, which is illustrated in Figure 15. For each sphere of radius $a, 20$ circular cone frustums with a height $\lambda_{\text {f,height }} a$ are uniformly distributed on it. The radius of the two bases equal $\lambda_{\text {bottom }} a$ and $\lambda_{\text {top }} a$, respectively. The distance between the sphere center and the top surface of a cone is $\lambda_{\text {p,height }} a$. Let $\lambda_{\mathrm{f} \text {,height }}=55 / 12, \lambda_{\text {bottom }}=33 / 125, \lambda_{\text {top }}=11 / 200$, and $\lambda_{\mathrm{p} \text {,height }}=11 / 2$. Therefore, parts of the frustums are inserted into the sphere. The volume of the above seaurchin-structured particle equals a sphere with a radius $a^{\prime}=1.1702 a$. Consider the rotation angle $\theta=69^{\circ}$ and $\kappa=3$.

We calculate the electrostatic forces $\tilde{F}_{A}$ for the twosphere case and the litchi structure using $\frac{F_{\mathcal{A}}}{4 \epsilon_{0} \epsilon_{f}\left(E_{0} a\right)^{2}}$ and $\frac{F_{\mathcal{A}}}{4 \epsilon_{0} \epsilon_{f}\left(E_{0} a^{\prime}\right)^{2}}$, respectively. As seen in Figure 16, unlike the litchi structure, the sea urchin structure contributes little to the electrostatic force and reduces the force: the force at the limit position of the sea urchin structure is $69 \%$ that of the two-sphere case. This is mainly because although this structure seems to be more closely integrated, in order to meet the assumption that no deformation occurs when the two particles are in contact with each other, the relative distance $\tau=d / a$ is quite limited (here $\tau$ should be larger than 3.35), which makes the force smaller than that in the two-sphere case where $\tau=2$.

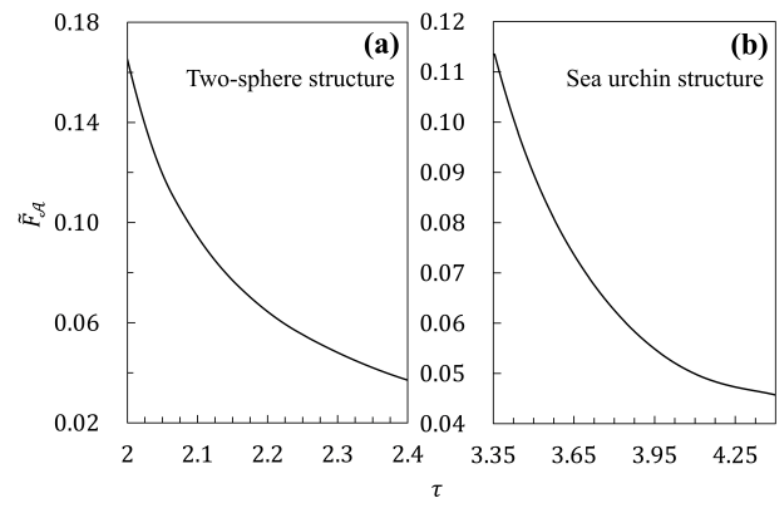

Figure 16. Electrostatic force (in units of $1 /\left(4 \epsilon_{0} \epsilon_{f} E_{0}^{2} a^{2}\right)$ for (a) the two-sphere structure and $1 /\left(4 \epsilon_{0} \epsilon_{f} E_{0}^{2} a^{\prime 2}\right)$ for (b) the sea urchin structure) as a function of spacing factor $\tau=d / a$ with $\kappa=3$ for (a) the two-sphere structure $v s$. (b) the sea urchin structure.

\section{Conclusions}

In this paper, we derive formulae of the electrostatic force between two spherical dielectric particles in the PD, DID, and MID models whose results are Eqs. (2.10), (3.7), and (3.8), respectively. The study of the convergence suggests that in the case of two identical spheres in MID models, the simplified version of Eq. (3.8), i.e., Eq. (4.2), is inapplicable when either of the following occurs (i) $\kappa>50$ and $\tau<2.1$, (ii) $\kappa>10$ and $\tau<2.03$, or (iii) $\kappa>4$ and $\tau<2.004$. The numerical simulation based on the finite element method (FEM) is then necessary, by which we conclude the following:

- In the n-sphere case, the electrostatic energy per sphere at the limit position is a function of $\kappa$. When $n$ is fixed, with the increase of $\kappa$, there exists a maximum energy.

- Compared to the case of having different particle sizes, the force between identical particles has the greatest force, which suggests making the particles as identical as possible in the experiment to increase the shear yield stress.

- Sub-structures uniformly grown on spherical particles can increase the force, but the effect depends on the balance of $\tau$ and the form of the sub-structures. A litchi structure is preferred.

Compared to the analytical methods, the FEM is a more efficient method to calculate the electrostatic force between particles, regardless of its form and relative permittivity. Thanks to this method, we could conceive a 
structure that can generate a greater force and create it in laboratory to obtain a better shear yield stress, which is applicable. Observed by an in situ electron microscope, we could build a 3D model of the particles and use the same method to calculate the force more accurately.

\section{Acknowledgements}

The project is fully supported by the personal savings of the corresponding author L. Gan who also undertook the bulk of the research. A great thanks to Y. H. Kwah, Y. T. Feng, G. Y. Mu, T. M. Nie, X. X. Han, and I. Zakia.

\section{References}

1. V. M. Zamudio, R. Nava, L. Rejón, M. A. Ponce, S. Víquez, and V. M. Castaño, Physica A: Statistical Mechanics and its Applications 227, 55 (1996).

2. S. Choi, C. C. Cheong, and G. Kim, Mechatronics 7, 53 (1997).

3. T. Kuriyagawa, M. Saeki, and K. Syoji, Precision Engineering 26, 370 (2002).

4. N. Stevens, J. Sproston, and R. Stanway, Journal of mechanisms, transmissions, and automation in design 110, 182 (1988).

5. Z. P. Shulman, R. G. Gorodkin, E. V. Korobko, and V. K. Gleb, Journal of Non-Newtonian Fluid Mechanics 8, 29 (1981).

6. A. Simmonds, in IEE Proceedings D-Control Theory and Applications (IET, 1991), pp. 400.

7. N. K. Petek, (SAE Technical Paper, 1992).

8. R. Stanway, J. Sproston, and A. El-Wahed, Smart Materials and Structures 5, 464 (1996).

9. K. Bohon, and S. Krause, Journal of Polymer Science-B-Polymer Physics Edition 36, 1091 (1998).

10. H. See, Journal of Industrial and Engineering Chemistry 10, 1132 (2004).

11. A. Lind, (FFI-rapport, 2008).

12. L. Marshall, C. F. Zukoski, and J. W. Goodwin, Journal of the Chemical Society, Faraday Transactions 1: Physical Chemistry in Condensed Phases 85, 2785 (1989).

13. M. Parthasarathy, and D. J. Klingenberg, Materials Science and Engineering: R: Reports 17, 57 (1996).

14. J. S. Marshall, and S. Li, Adhesive particle flow (Cambridge University Press, 2014).

15. K. Barros, and E. Luijten, Physical Review Letters 113, 017801 (2014).

16. K. Barros, D. Sinkovits, and E. Luijten, Journal of Chemical Physics 140, 064903 (2014).

17. J. M. Kosterlitz, and D. J. Thouless, Journal of Physics C: Solid State Physics 6, 1181 (1973).

18. R. Tao, J. Woestman, and N. Jaggi, Applied Physics Letters 55, 1844 (1989).

19. W. Wen, X. Huang, and P. Sheng, Soft Matter 4, 200 (2008).

20. S. Gawade, and A. Jadhav, International Journal of Engineering Research \& Technology 1, 1 (2012).

21. A. Goyette, and A. Navon, Physical Review B 13, 4320 (1976).
22. R. Tao, Q. Jiang, and H. Sim, Physical Review E 52, 2727 (1995).

23. Y. Siu, J. T. Wan, and K. Yu, Computer Physics Communications 142, 446 (2001).

24. J. D. Jackson, Classical Electrodynamics, 3rd ed. (Wiley, 1999).

25. J. N. Foulc, P. Atten, and N. Felici, Journal of electrostatics 33, 103 (1994).

26. K. Ahn, and D. J. Klingenberg, Journal of Rheology 38, 713 (1994).

27. J. Cao, J. Huang, and L. Zhou, Journal of Physical Chemistry B 110, 11635 (2006).

28. J. M. Jin, The Finite Element Method in Electromagnetics, 3rd ed. (Wiley, 2015).

29. D. F. Watson, The Computer Journal 24, 167 (1981).

30. A. Bowyer, The Computer Journal 24, 162 (1981).

31. P. Vaněk, J. Mandel, and M. Brezina, Computing 56, 179 (1996).

32. M. Sibuya, Annals of the Institute of Statistical Mathematics 14, 81 (1962).

33. R. L. Smith, Operations Research 32, 1296 (1984).

34. E. B. Saff, and A. B. Kuijlaars, The Mathematical Intelligencer 19, 5 (1997). 